\title{
ROTUNDITY, ORTHOGONALITY, AND CHARACTERIZATIONS OF INNER PRODUCT SPACES
}

\author{
BY J. R. HOLUB 1 \\ Communicated by Alston Householder, May 30, 1975
}

1. The purpose of this paper is to announce a number of interesting new results concerning the geometry of normed linear spaces. In particular, we present some new characterizations of rotund normed linear spaces and of inner product spaces. The general theme connecting the two topics is the realization that special cases of conditions characterizing inner product spaces are often in themselves characterizations of rotundity.

Details and proofs will appear elsewhere.

Throughout the paper $E$ will denote a real normed linear space (n.l.s.) and $M$ a subspace of $E$, denoted $M \subset E$. If $\left\{x_{i}\right\}_{i=1}^{n}$ is a subset of $E,\left[x_{i}\right]_{i=1}^{n}$ will denote the linear span of $\left\{x_{i}\right\}$.

2. Rotundity. Recall that a n.l.s. $E$ is said to be rotund [3] (or strictly convex [2]) if every point on the unit sphere in $E$ is an extreme point. Our first result shows that rotundity is characterized by a very desirable condition involving the cone in $E$ generated by a set of vectors.

DEFINITION 1. Let $\left\{x_{i}\right\}_{i=1}^{2}$ be a normalized linearly independent pair of vectors in $E$. Then $C\left\{x_{i}\right\}=\left\{a_{1} x_{1}+a_{2} x_{2} \mid a_{1} \cdot a_{2} \geqslant 0\right\}$ is called the cone of $\left\{x_{i}\right\}_{i=1}^{2}$ in $E$.

THEOREM 1. E is rotund $\Leftrightarrow$ for any normalized linearly independent set $\left\{x_{i}\right\}_{i=1}^{2}$ in $E$, the set of points in $\left[x_{i}\right]_{i=1}^{2}$ equidistant from $x_{1}$ and $x_{2}$ is a subset of $C\left\{x_{i}\right\}$.

Another characterization of rotundity which has a similar flavor is based on the following lemma which is interesting in its own right.

Lemma 1. Let $E$ be a 2-dimensional n.l.s. Then every point $x \in E$ with $\|x\|<1$ is the midpoint of a chord of the unit sphere in $E$ (i.e. there exist $x_{1}$ and $x_{2}$ with $\left\|x_{1}\right\|=\left\|x_{2}\right\|=1$ for which $\left.x=(1-\lambda) x_{1}+\lambda x_{2}, 0<\lambda<1\right)$.

A natural question concerns the uniqueness of such a chord (for $x \neq 0$ ). The answer is given by

AMS (MOS) subject classifications (1970). Primary 46B99, 46C05, 46C10.

${ }^{1}$ Research partially supported by NSF grant GP 33778 . 
THEOREM 2. $E$ is rotund $\Leftrightarrow$ given any two-dimensional subspace $M \subset E$ and any $x \in M$ with $0<\|x\|<1$, then $x$ is the midpoint of a unique chord of the unit sphere in $M$.

Another result of a similar nature is

THEOREM 3. $E$ is rotund $\Leftrightarrow$ given any two-dimensional subspace $M \subset E$ and any $x \in M$ with $0<\|x\| \leqslant 1$ there exists a unique pair of normalized vectors $x_{1}$ and $x_{2}$ in $M$ for which $x=x_{1}+x_{2}$.

A final result links rotundity to the number of linearly independent points which norm a projection on $E$.

Definition 2. If $E$ and $F$ are n.l.s. and $T: E \rightarrow F$ a bounded linear operator, a point $x \in E$ for which $\|x\|=1$ and $\|T x\|=\|T\|$ is called a norming point of $T$.

THEOREM 4. $E$ is rotund $\Leftrightarrow$ given any n-dimensional subspace $M \subset E \quad(1 \leqslant$ $n<+\infty)$ and any projection $P: E \rightarrow M$, then $P$ has at most $n$ linearly independent norming points.

3. Orthogonality and inner product spaces. Several (nonequivalent) definitions of orthogonality in n.l.s. have been given by various authors. Two of these are

DEFINITION 3 (BIRKHOFF [1]). $x \perp_{B} y \Leftrightarrow\|x+\lambda y\| \geqslant\|x\|$ for all $\lambda$.

Definition 4 (JAmes [4]). $x \perp_{J} y \Leftrightarrow\|x+y\|=\|x-y\|$.

Interesting results concerning the characterization of inner product spaces through the assumption of additional properties of $B$-orthogonality (Definition 3 ) have been obtained by Birkhoff [1] and James [5], [6]. We continue this study in the following direction.

In an inner product space, if $y \in\left[x_{i}\right]_{i=1}^{n}$ and $y \perp x_{i}$ for all $i$, then $y=0$. We show that this situation essentially characterizes inner product spaces with respect to both $B$-orthogonality and $J$-orthogonality.

THEOREM 5. $E$ is an inner product space $\Leftrightarrow$ whenever $\left\{x_{i}\right\}_{i=1}^{n}$ is a linearly independent set of normalized vectors in $E(n \geqslant 3)$ and $y \in\left[x_{i}\right]_{i=1}^{n}$ with $y \perp_{B} x_{i}$ for all $i$, then $y=0$.

COROLlaRY 1. E is an inner product space $\Leftrightarrow$ given $\left\{x_{i}\right\}_{i=1}^{n}$ a linearly independent normalized set in $E(n \geqslant 3)$ and $\left\{x_{i}^{*}\right\}_{i=1}^{n} \subset E^{*}$ with $\left\|x_{i}^{*}\right\|=1=$ $\left\langle x_{i}^{*}, x_{i}\right\rangle$ for all $i$, then $\operatorname{det}\left[\left\langle x_{i}^{*}, x_{j}\right\rangle\right] \neq 0$.

Note. If in Theorem 5 we restrict ourselves to sets of two vectors, another characterization of rotundity is obtained.

For the case of $J$-orthogonality we have

THEOREM 6. E is an inner product space $\Leftrightarrow$ whenever $\left\{x_{i}\right\}_{i=1}^{n}$ is a linearly 
independent normalized set in $E(n \geqslant 2)$ and $y \in\left[x_{i}\right]_{i=1}^{n}$ with $y \perp_{J} x_{i}$ for all $i$, then $y=0$.

Finally we have two more straightforward characterizations.

THEOREM 7. $E$ is an inner product space $\Leftrightarrow$ whenever $\left\|x_{1}\right\|=\left\|x_{2}\right\|$, then $x_{1}+x_{2} \perp_{B} x_{1}-x_{2}$.

THEOREM 8. $E$ is an inner product space $\Leftrightarrow$ whenever $x \perp_{B} y$ then $x \perp_{J} y$.

Note. In regard to Theorem 8, Day has shown that $E$ is an inner product space $\Leftrightarrow$ whenever $x \perp_{J} y$ then $x \perp_{B} y[3]$.

\section{REFERENCES}

1. G. Birkhoff, Orthogonality in linear metric spaces, Duke Math. J. 1 (1935), 169172.

2. J. Clarkson, Uniformly convex spaces, Trans. Amer. Math. Soc. 40 (1936), 396414.

3. M. M. Day, Normed linear spaces, Ergebnisse der Mathematik und ihrer Grenzgebiete, N. F., Heft 21, Academic Press, New York; Springer-Verlag, Berlin, 1962. MR 26 \#2847. 4. R. C. James, Orthogonality in normed linear spaces, Duke Math. J. 12 (1945), 291302. MR 6, 273.

5. - Orthogonality and linear functionals in normed linear spaces, Trans. Amer. Math. Soc. 61 (1947), 265-292. MR 9, 42.

6. Inner products in normed linear spaces, Bull. Amer. Math. Soc. 53 (1947), 559-566. MR 9, 42.

DEPARTMENT OF MATHEMATICS, VIRGINIA POLYTECHNIC INSTITUTE AND STATE UNIVERSITY, BLACKSBURG, VIRGINIA 24061 